\title{
La distribución de recursos entre Comunidades Autónomas. Una primera aproximación a los cambios derivados del nuevo modelo de financiación
}

\author{
LUIS ÁNGEL HIERRO RECIO, PEDRO ATIENZA MONTERO y \\ ROSARIO GÓMEZ-ÁLVAREZ DÍAZ \\ Departamento de Economía e Historia Económica \\ UNIVERSIDAD DE SEVILLA \\ e-mail: 1hierro@us.es; atienza@us.es; charogomez@us.es
}

\begin{abstract}
RESUMEN
El presente trabajo tiene como objetivo analizar el cambio en los resultados distributivos del nuevo modelo de financiación autonómica de régimen común con respecto al vigente desde 2002. Para ello se utilizan indicadores de desigualdad y progresividad derivados de curvas de concentración, así como indicadores de dispersión y de elasticidad. El resultado fundamental que se obtiene es que, a pesar de las modificaciones sustanciales que se producen en la estructura del nuevo modelo, ello no conlleva cambios significativos en los efectos redistributivos de las transferencias, ni en términos de desigualdad ni en términos de progresividad. No obstante, el nuevo modelo sí produce menos reordenación y, en consecuencia, menos desigualdad generada por la misma.

Palabras clave: Financiación autonómica, federalismo fiscal, desigualdad, progresividad, transferencias intergubernamentales, análisis comparado.
\end{abstract}

\section{The Distribution of Resources between Autonomous Communities. A First Approximation to the Changes Derived from the New Model of Financing}

\begin{abstract}
The present work has as aim to analyze the change in the distributive results of the new model of financing of Autonomous Communities of common regime with regard to model in force from 2002. For it we use indicators of inequality and progressiveness derived from curves of concentration, as well as indicators of dispersion and of elasticity. The fundamental result obtained is that, in spite of the substantial modifications that are produced in the structure of the new model, it does not carry significant changes in the redistributive effects of the transfers, nor in terms of inequality nor in terms of progressiveness. Nevertheless, the new model produces less reranking and, in consequence, less inequality generated by the same one.
\end{abstract}

Keywords: Spanish System of Regional Financing, Fiscal Federalism, Inequality, Progressiveness, Intergovernmental Grants, Compared Research.

Clasificación JEL: H71, H73. 


\section{INTRODUCCIÓN Y OBJETIVOS}

El presente trabajo tiene por objeto conocer el impacto que puede tener la reforma del sistema de financiación autonómica de 2009 sobre la distribución de recursos financieros entre las CC.AA. mediante la comparación entre los resultados distributivos del modelo de financiación autonómica de régimen común vigente desde 2002 con el acordado en el Consejo de Política Fiscal y Financiera (Acuerdo 6/2009 de 15 de julio). Para ello y siguiendo la estela marcada por los recientes trabajos sobre desigualdad y distribución de recursos financieros de Martínez-Vázquez y Boex (1999), Rao (2000), Bird y Tarasov (2002), Brodjonegoro, Martínez-Vázquez (2002), Hofman y Cordeira (2004) e Hierro, Atienza y Patiño (2007) e Hierro, Atienza y Gómez-Álvarez (2008) vamos a utilizar una serie de indicadores de desigualdad y de progresividad que nos permita valorar la distribución de ingresos resultante en cada modelo. Así, nos proponemos medir la dispersión de resultados financieros per cápita y su desviación en relación a la isodistribución de forma que podamos determinar cuánto modifica la distribución el sistema de transferencias del modelo 2009 respecto al vigente desde 2002. Igualmente nos proponemos medir la relación entre los resultados distributivos y los niveles de producción de las CC.AA. a fin de conocer en qué medida la distribución de renta y producción condiciona la distribución de recursos entre las CC.AA. y si las transferencias contribuyen a alterar significativamente el sentido de la distribución. Finalmente realizaremos algunas apreciaciones sobre la reordenación que producen las transferencias en ambos modelos.

El problema fundamental con el que nos enfrentamos para conseguir nuestro objetivo es la ausencia de un cuadro oficial de ingresos para el año base. Dada esta carencia tomaremos como referencia las estimaciones realizadas en De la Fuente (2009a, 2009b). Dichas estimaciones han sido revisadas por el propio autor en De la Fuente (2009c) durante la tramitación de la Ley 22/2009, por la que se regula el sistema de financiación de las Comunidades Autónomas de régimen común y Ciudades con Estatuto de Autonomía y se modifican determinadas normas tributarias, y de la Ley Orgánica 3/2009, de modificación de la Ley Orgánica 8/1980, de 22 de septiembre, de Financiación de las Comunidades Autónomas. La revisión se basa en que el articulado de la ley 22/2009 prevé un recálculo de las cantidades del modelo para 2009 con el fin de evitar que el impacto de la crisis económica en la recaudación dé lugar a efectos distorsionantes sobre las cantidades previstas. No obstante, dado lo estimativo de cualquier cifra del ejercicio que se acaba de cerrar, preferimos utilizar las cifras calculadas para el año 2007, que además son las que al fin y al cabo revelan la verdadera intención de la reforma del modelo.

Para llevar a cabo nuestra tarea estructuramos el trabajo de la siguiente forma. En primer lugar se describe la metodología de medición a utilizar, a continuación se describen someramente los modelos aprobados en 2001 y 2009 y se presentan las cifras. Posteriormente se calcularán los indicadores representativos de la desigualdad y la progresividad en la distribución de los recursos antes y después de transferencias y se analizará la información que nos aportan, finalizando con un apartado de conclusiones. 


\section{INDICADORES A APLICAR}

Para el objetivo que nos proponemos en el presente trabajo vamos a utilizar los siguientes indicadores:

- Coeficiente de variación. Comúnmente utilizado en trabajos de este tipo, mide la dispersión de la distribución con respecto a la media. Su ecuación es, respectivamente para los ingresos antes y después de transferencias:

$$
\begin{gathered}
C V_{X a}=\frac{S_{X a}}{\mu_{a}} \\
C V_{X d}=\frac{S_{X d}}{\mu_{d}}
\end{gathered}
$$

donde $S_{x a}$ y $S_{x d}$ son la desviación típica antes y después de transferencias y $\mu_{a} \mu_{d}$ los ingresos medios per cápita para el conjunto de CC.AA. antes y después de transferencias, respectivamente.

La disminución de la dispersión derivada de la incorporación de las transferencias (reducción relativa del coeficiente de variación) podemos estimarla como:

$$
\frac{C V_{X a}-C V_{X d}}{C V_{X a}} \times 100
$$

- El índice de concentración de los ingresos autonómicos antes y después de transferencias. Es el cociente entre el área que dista entre la línea de equidistribución y la curva de concentración de los recursos de las CC.AA., ordenadas de menor a mayor ingreso per cápita en cada caso. Dichos índices de concentración antes y después de transferencias vendrían dados por:

$$
\begin{aligned}
& C\left(x_{a}\right)=1-2 \int_{0}^{1} L x_{a}(p) d p \\
& C\left(x_{d}\right)=1-2 \int_{0}^{1} L x_{d}(p) d p
\end{aligned}
$$

siendo $\operatorname{Lxa}(p)$ y $\operatorname{Lxd}(p)$ las funciones representativas de la curvas de concentración de los ingresos de las CC.AA. antes y después de transferencias.

- Índices de Reynolds-Smolenski (1977) y de Pechman-Okner (1984). Los mismos vendrían dados por:

$$
\begin{aligned}
& R S(x)=C(x a)-C(x d) \\
& P O(x)=\frac{C(x a)-C(x d)}{C(x a)} \times 100
\end{aligned}
$$


- Impacto Reordenador. A fin de que nuestra perspectiva sea completa también podemos derivar de los índices de concentración una medida de la reordenación. Cuando el conjunto de transferencias hace que una C.A. supere a otra en el orden de ingresos lo que ha ocurrido es que la desigualdad que antes existía por tener menos ingresos ahora existe porque tiene más. Un sistema de financiación que produzca poca reordenación nos indicará que los criterios de distribución de recursos tienden a consolidar las situaciones de partida, mientras que si la reordenación es alta se estarán utilizando criterios de reparto que distan de los implícitos en la distribución de recursos tributarios.

Para medir la reordenación podemos utilizar el método de Kakwani (1984) después ampliado por Aronson, Johnson y Lambert (1994). Así, si denominamos $\bar{C}\left(x_{d}\right)$ al índice de concentración de los ingresos después de transferencias, calculado a partir de la ordenación que generan los recursos antes de transferencias (los ingresos tributarios) y deducimos el índice de concentración de los ingresos totales obtendremos la desigualdad generada por la reordenación. Si esta cifra la dividimos por el índice de concentración de los ingresos tributarios (antes de transferencias) nos dará un indicador relativo de cuánta reordenación genera el sistema $(I R r)$.

$$
\operatorname{IRr}(x)=\frac{\bar{C}\left(x_{d}\right)-C\left(x_{d}\right)}{C\left(x_{a}\right)}
$$

Expresado en tanto por ciento nos indica cuanta desigualdad de nuevo cuño, producida por la reordenación, ha sustituido a la desigualdad original.

- Elasticidad renta de las transferencias. Estimando una regresión lineal en logaritmos tal como recoge la siguiente expresión:

$$
\operatorname{LgTRp}_{i}=\alpha+\beta \cdot \operatorname{LgPIBp} c_{i}+u_{i}
$$

donde $T R p c_{i}$ y $P I B p c_{i}$ son las transferencias per cápita y el PIB per cápita de las $i$ CC.AA., la estimación de $\beta$ es un indicador de la elasticidad renta de la transferencias.

- Índice de Suits (1977). Si denominamos $\operatorname{Lxa}(y)$ y $\operatorname{Lxd}(y)$ a las funciones representativas de la curvas de concentración relativas de los ingresos de las CC.AA. antes y después de transferencias, que indica el porcentaje de ingresos acumulado por las CC.AA. que acumulan el $y$ por ciento de la renta total del país, entonces a partir de esas funciones continuas se definen los respectivos índices de Suits $(S)$ como:

$$
\begin{aligned}
& S\left(x_{a}\right)=1-2 \int_{0}^{1} L x_{a}(y) d y \\
& S\left(x_{d}\right)=1-2 \int_{0}^{1} L x_{d}(y) d y
\end{aligned}
$$


- Índice de Kakwani (1977). Si $G(y)$ es el Gini del PIB per cápita de las CC.AA., el índice de Kakwani $(K)$ antes y después de transferencias será respectivamente:

$$
\begin{aligned}
& K(x a)=C(x a)-G(y) \\
& K(x d)=C(x d)-G(y)
\end{aligned}
$$

Los índices de Suits y Kakwani y la estimación de la elasticidad renta de las transferencias son indicativos del sentido de la distribución, de forma que podemos valorar si los recursos antes y después de transferencias se orientan hacia las CC.AA. con mayor o menor nivel de producción. Miden, podemos decir, el grado de progresividad con que se distribuyen los recursos.

\section{LOS MODELOS DE FINANCIACIÓN AUTONÓMICA 2002 Y 2009}

Las características básicas de los dos modelos de financiación son las que a continuación se describen.

\subsection{Modelo 2002}

El modelo de financiación autonómica de régimen común vigente desde $2002^{1}$ mantiene un modus operandi en la configuración de su estructura financiera similar a la de los anteriores modelos. Así, se parte de una restricción financiera inicial para un año base, 1999, distinguiendo tres bloques de financiación, las de competencias comunes, sanidad y servicios sociales y el montante de dicha restricción se distribuye entre las CC.AA. según distintos indicadores, donde la población tiene su papel preponderante y de manera que esa distribución no suponga una financiación a cada C.A. por debajo de la restricción mínima antes establecida, es decir, como siempre, todas las CC.AA. han de salir ganando en los resultados financieros obtenidos (principio de neutralidad financiera). Concretamente, el bloque de financiación de las competencias comunes ${ }^{2}$ se distribuye según las siguientes variables: población, con un peso del $94 \%$, superficie, $4,2 \%$, dispersión, $1,2 \%$ e insularidad $0,6 \%$. Por su parte, las variables de reparto del bloque de financiación de los servi-

\footnotetext{
${ }^{1}$ Regulado por L.O. 3/1996, Ley 21/2001, Ley 7/2001 de modificación parcial de LOFCA y Acuerdo del CPFF de 27 de julio de 2001. Para una descripción pormenorizada véase De la Fuente y Gundín (2008) o Atienza (2003), entre otros.

${ }^{2}$ Más bien el Fondo General de dicho bloque, por que también se crean dos específicos: Fondo específico de Renta Relativa y otro "para paliar la escasa densidad de población" (véase Atienza (2003) o De la Fuente y Gundín (2008)). Dicho Fondo General queda integrado, en valores del año base 1999, primero, por las liquidaciones definitivas en dicho año en concepto de la tarifa autonómica del IRPF, participación en Ingresos del Estado, tanto de su tramo general como del particular de participación en la recaudación territorial del IRPF y el Fondo de Garantía. En segundo lugar, por la recaudación normativa de tributos cedidos y tasas afectas a servicios traspasados.
} 
cios sanitarios son la población protegida, con una ponderación del $75 \%$, población mayor de 65 años, con el $24,5 \%$ y por el hecho insular, $0,5 \%$. Por último el bloque referido a los servicios sociales se distribuye en exclusiva según la variable población mayor de 65 años $^{3}$. Por otra parte, los recursos del sistema que han de financiar esa cuantía inicial están compuestos por las tasas cedidas y los tributos cedidos tradicionales, sobre los que se amplía la capacidad normativa ${ }^{4}$, la cesión parcial del IRPF, ahora en un porcentaje del 33\% y con facultades normativas ampliadas en materia de tarifa y deducciones, y nuevos tributos, como el IVA y los Impuestos Especiales, sin cesión de capacidad normativa alguna, sobre los cuales se cede un porcentaje, $35 \%$ y $40 \%$ respectivamente, de su recaudación, la cual se distribuye territorialmente en base a indicadores de consumo de cada C. $A^{5}$. La anterior PIE es sustituida por el Fondo de Suficiencia, el cual sigue funcionando como mecanismo de cierre del sistema y que se cuantifica como la diferencia entre la cuantía de la restricción inicial para cada C.A. y los montantes que representan las recaudaciones normativas por tributos cedidos, y la cuantía que suponen la cesión parcial del IRPF y los nuevos recursos por IVA e Impuestos Especiales para el año 1999. Ahora la novedad consiste en que el Fondo de Suficiencia no se establece como el producto de un porcentaje sobre una base (anteriormente ITAE), como en la anterior PIE, sino como una cantidad monetaria para el año base 1999 que se hace evolucionar según los denominados Ingresos Tributarios del Estado (ITE).

\subsection{Modelo 2009}

Respecto al modelo aprobado en $2009^{6}$, como en la configuración de los modelos anteriores, se establecen las necesidades globales de financiación para ese año, como la suma de los resultados del modelo anterior para el año base, 2007, y algunos recursos adicionales aportados por el Estado que se reparten fundamentalmente en proporción al aumento de la población ajustada entre 1999 y 2007. Por otra parte, se estima la recaudación normativa para 2007 mediante una actualización de la existente en 1999 y añadiéndole el incremento de recaudación normativa proveniente de los nuevos porcentajes de cesión de tributos que establece este nuevo

\footnotetext{
${ }^{3}$ Ambos bloques de financiación de servicios sanitarios y sociales se financian según la liquidación definitiva de la financiación de los mismos en el año base 1999.

${ }^{4}$ Impuesto sobre el Patrimonio, sobre Transmisiones Patrimoniales, sobre Actos Jurídicos Documentados, sobre Sucesiones y Donaciones y las Tasas sobre el Juego.

${ }^{5}$ También se ceden por primera vez nuevos tributos como el Impuesto Especial sobre Determinados Medios de Transporte y el Impuesto sobre las Ventas Minoristas de Determinados Hidrocarburos. Se cede tanto el rendimiento recaudatorio total como ciertas facultades normativas. Y por otra parte se cede una participación territorializada (sin competencia normativa alguna) del $100 \%$ de la recaudación líquida por el Impuesto sobre la Electricidad.

${ }^{6}$ Regulado por Acuerdo del CPFF 6/2009 de 15-7-2009, por la Ley 22/2009, de 18 de diciembre, por la que se regula el sistema de financiación de las Comunidades Autónomas de régimen común y Ciudades con Estatuto de Autonomía y se modifican determinadas normas tributarias y por Ley Orgánica 3/2009, de 18 de diciembre, de modificación de la Ley Orgánica 8/1980, de 22 de septiembre, de Financiación de las Comunidades Autónomas.
} 
modelo ${ }^{7}$. De este total de ingresos tributarios normativos cada C.A. debe aportar un 75\% para dotar el nuevo Fondo de Garantía de Servicios Públicos Fundamentales, constituyendo, por tanto, el $25 \%$ restante de recaudación tributaria normativa más la recaudación real extra sobre ésta última que consiga realizar cada C.A. en base a su mayor esfuerzo fiscal los ingresos tributarios reales de cada C.A.

En cuanto a las transferencias el modelo establece tres. Primero, el ya mencionado Fondo de Garantía de Servicios Públicos Fundamentales, que se financia, además del $75 \%$ de recaudación normativa de cada C.A., con unos recursos adicionales aportados por el Estado (y actualizados por los ITE). Los criterios de reparto del Fondo cada año se basan en una estimación de las necesidades de gasto en la provisión de los servicios públicos fundamentales, y donde los indicadores principales son la población, que alcanza un peso del $30 \%$, la población entre $0 \mathrm{y}$ 16 años el 20,5\%, la población mayor de 65 años el 8,5\% y la población protegida equivalente el $38 \%$ (lo que da lugar al concepto población ajustada) ${ }^{8}$. El segundo lugar, el Fondo de Suficiencia Global, que, al igual que en el modelo anterior se calcula de forma residual para el año base para cada C.A., ahora como la diferencia entre las necesidades globales de financiación para ese año y la suma entre el Fondo de Garantía de Servicios Públicos Fundamentales y el 25\% de la recaudación normativa de tributos de cada C.A. A partir de este año base, la dotación para los años siguiente se realiza en función de una misma tasa de variación para todas las CC.AA., los ITE (tramos no cedidos del IRPF, IVA e Impuestos Especiales). En tercer lugar, los Fondos de Convergencia autonómica, que se financian mediante recursos adicionales del Estado. Se trata de dos Fondos: el Fondo de Cooperación y el Fondo de Competitividad. El primero tiene como objetivo promover la convergencia real de las CC.AA. favoreciendo a las CC.AA. con menor renta per cápita. De esta manera las beneficiarias serán aquella cuyo PIBpc sea inferior al $90 \%$ del conjunto de CC.AA. de régimen común; en segundo lugar, aquellas cuya densidad de población sea inferior al $50 \%$ de la densidad media del mismo conjunto de CC.AA.; y por último, aquellas, que teniendo un crecimiento de población inferior al $90 \%$ de la media correspondiente a las Comunidades Autónomas de régimen común, tengan una densidad de población por kilómetro cuadrado inferior a la cifra resultante de multiplicar por 1,25 la densidad media de las mencionadas Comunidades Autónomas de régimen común. Por su parte, el Fondo de Competitividad se repartirá anualmente entre las Comunidades Autónomas de régimen común con financiación per cápita ajustada inferior a la media o a su capacidad fiscal, en función de su población ajustada relativa.

Los dos modelos de financiación autonómica que se acaban de describir someramente dan como resultado una financiación de las CC.AA. de régimen común como la que se recoge en la Tabla 1 . Se ha tomado el año 2007 para realizar la

\footnotetext{
${ }^{7}$ Se eleva del 33\% al 50\% la cesión parcial en el IRPF, del 40\% al 50\% en el IVA y hasta el 58\% en los Impuestos Especiales de Fabricación. Véase Acuerdo del CPFF del 15-7-2009 y ley 22/2009.

${ }^{8}$ El resto de variables de reparto son la superficie, la dispersión y la insularidad, con unas ponderaciones respectivas de $1,8 \%, 0,6 \%$ y $0,6 \%$ respectivamente.
} 
comparación de los dos modelos, primero, por que es el último año del modelo 2002, cuyos datos liquidados están publicados por el Ministerio de Economía y Hacienda; segundo, porque para ese año se dispone de una estimación realizada por De la Fuente $(2009$ a, 2009b) y ante la carencia de datos oficiales es la única existente; tercero, porque como se ha señalado más arriba originalmente el año 2007 es el año base del modelo aprobado en el CPFF en julio de 2009; cuarto, porque la Ley 22/2009 prevé una actualización de las cifras tomando como referencia el año 2009, a fin de considerar los efectos de la crisis económica, entendemos que la ausencia de datos liquidados hacen menos fiables las estimaciones; y quinto, porque si en el ánimo de los negociadores está que el modelo dé como resultado los estimados para 2007 parece razonable que los datos que finalmente se obtengan para 2009 no sean los determinantes a largo plazo.

Para lograr la máxima homogeneización asumimos también los ajustes realizados en De la Fuente (2009a, 2009b). Estos ajustes son, en primer lugar, estimar el Fondo de Suficiencia Global a competencias homogéneas y, segundo, ajustar la recaudación tributaria de Canarias, ya que, al disponer de un Régimen Económico y Fiscal propio (REF), no participa en la recaudación del IVA ni de algunos Impuestos Especiales. Por tanto, De la Fuente realiza la homogeneización tributaria de esta C.A. incluyendo los recursos de esta C.A. por su participación en REF.

Entre los ingresos tributarios sólo se incluyen los tributos cedidos y tasas afectas a servicios traspasados a recaudación normativa, excluyendo los tributos propios y los recargos sobre tributos estatales. De esta manera las medidas de desigualdad reflejan un esfuerzo fiscal estándar para todas las CC.AA. y no incorporan la recaudación adicional derivada del uso que cada C.A. hubiere hecho de su capacidad normativa en las distintas figuras tributarias.

Respecto a las transferencias se incluyen sólo las incorporadas a la financiación ordinaria e incondicionada de los servicios públicos competencia de las CC.AA., con unos objetivos de suficiencia financiera y de nivelación de las necesidades de gasto. De esta forma se han incluido, en el modelo 2002 el Fondo de Suficiencia a competencias homogéneas, el Fondo de Garantía sanitaria y la dotación complementaria para la asistencia sanitaria establecida en la Segunda Conferencias de Presidentes. En el modelo 2009 se incluyen: el Fondo de Garantía de Servicios Públicos Fundamentales, el Fondo de Suficiencia Global y los Fondos de Convergencia: Fondo de Competitividad y Fondo de Cooperación. 


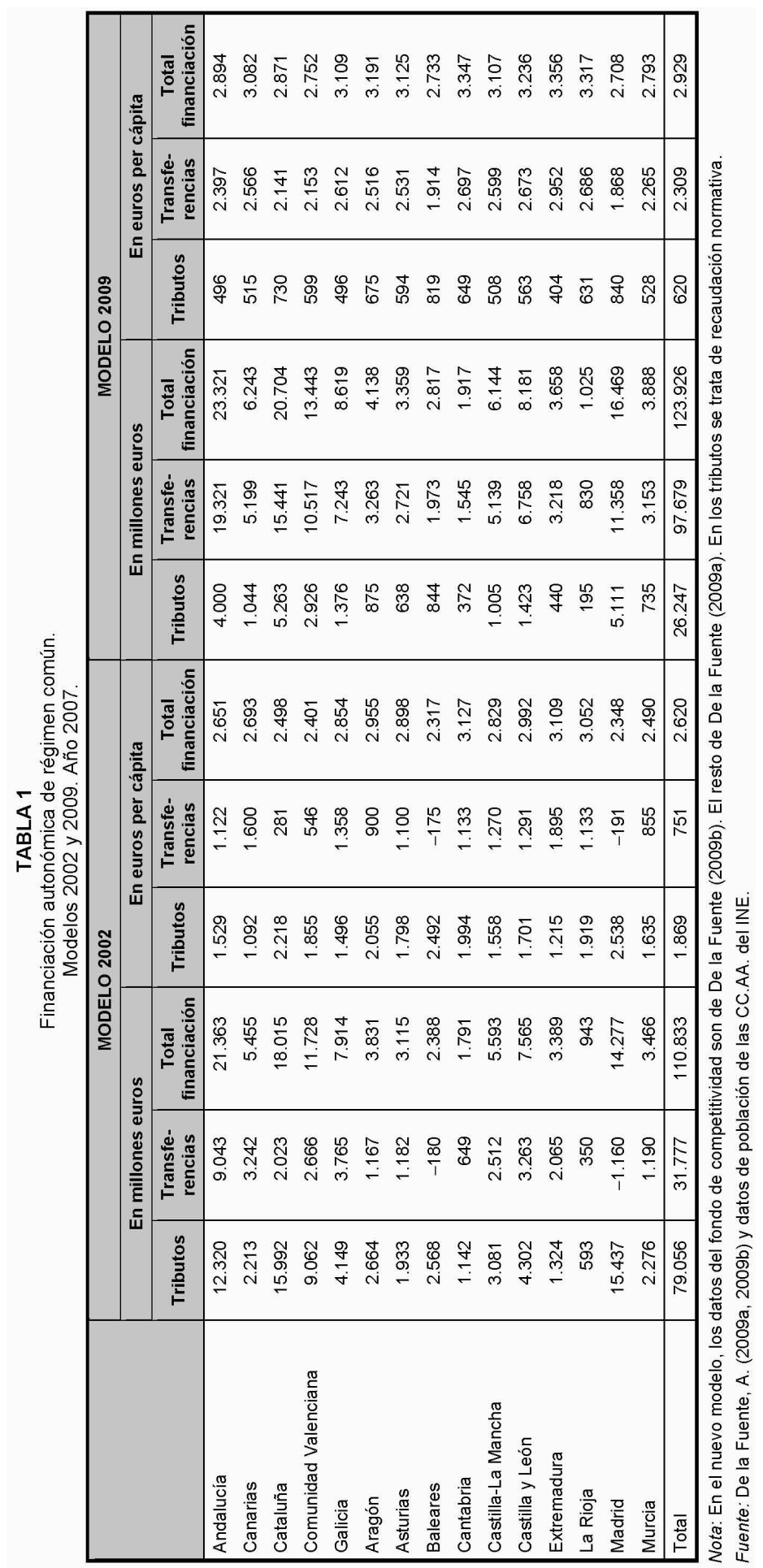




\section{LOS RESULTADOS}

En la Tabla 2 se exponen los resultados de los cálculos de los distintos indicadores utilizados para medir el impacto redistributivo de las transferencias.

En la situación antes de transferencias, los dos indicadores de desigualdad, coeficiente de variación e índice de concentración, manifiestan que el nuevo modelo aumenta, aunque de forma leve, la igualdad con que se distribuyen los recursos tributarios. Dado que hemos utilizado estimaciones de recaudación normativa, las cifras están depuradas del ejercicio de la capacidad normativa por parte de las CC.AA., por lo que la explicación a este aumento de la igualdad se encuentra en que el efecto del incremento de los porcentajes de cesión de los tributos del nuevo modelo es de una menor desigualdad en la distribución de las bases impositivas cedidas.

TABLA 2

Indicadores de distribución de recursos según el modelo 2002 y el nuevo modelo.

Estimaciones para el año 2007.

\begin{tabular}{|c|c|c|c|}
\hline \multicolumn{2}{|l|}{ Indicadores de Desigualdad } & Modelo 2002 & Nuevo modelo \\
\hline \multirow{2}{*}{ Coeficiente variación } & $T$ & 0,2239 & 0,1982 \\
\hline & $T+T R$ & 0,0873 & 0,0635 \\
\hline $\begin{array}{l}\text { Reducción relativa del coeficiente de } \\
\text { variación }\end{array}$ & & $61,03 \%$ & $67,98 \%$ \\
\hline \multirow{2}{*}{ Índice de Concentración } & $T$ & 0,1259 & 0,1161 \\
\hline & $T+T R$ & 0,0489 & 0,0349 \\
\hline Índice de Reynolds-Smolensky & & 0,0769 & 0,0812 \\
\hline Índice de Pechman-Okner & & $61,13 \%$ & $69,92 \%$ \\
\hline Contribución por reordenación & & $-0,0797$ & $-0,0543$ \\
\hline Reordenación relativa & & $-63,30 \%$ & $-46,79 \%$ \\
\hline \multicolumn{2}{|l|}{ Indicadores de Progresividad } & Modelo 2002 & Nuevo modelo \\
\hline \multirow{3}{*}{ Elasticidad renta de las transferencias } & Elasticidad & $-1,4201$ & $-0,4424$ \\
\hline & $t$-Student & $-1,976$ & $-2,651$ \\
\hline & $p$-valor & 0,074 & 0,02 \\
\hline \multirow{2}{*}{ Índice de Suits } & $T$ & 0,0024 & 0,0010 \\
\hline & $T+T R$ & $-0,1364$ & $-0,1261$ \\
\hline \multirow{2}{*}{ Índice de Kakwani } & $T$ & 0,0019 & 0,0009 \\
\hline & $T+T R$ & $-0,1356$ & $-0,1252$ \\
\hline
\end{tabular}

Nota: $T$ : total de ingresos tributarios; $T R$ : total de ingresos por transferencias.

Fuente: Elaboración propia a partir de los datos de la Tabla 1. 
Las curvas de concentración de la Figura 1 nos permiten deducir que la mejora leve en la igualdad se produce en las CC.AA. de menores recursos tributarios per cápita.

FIGURA 1

Curvas de concentración de ingresos tributarios normativos.

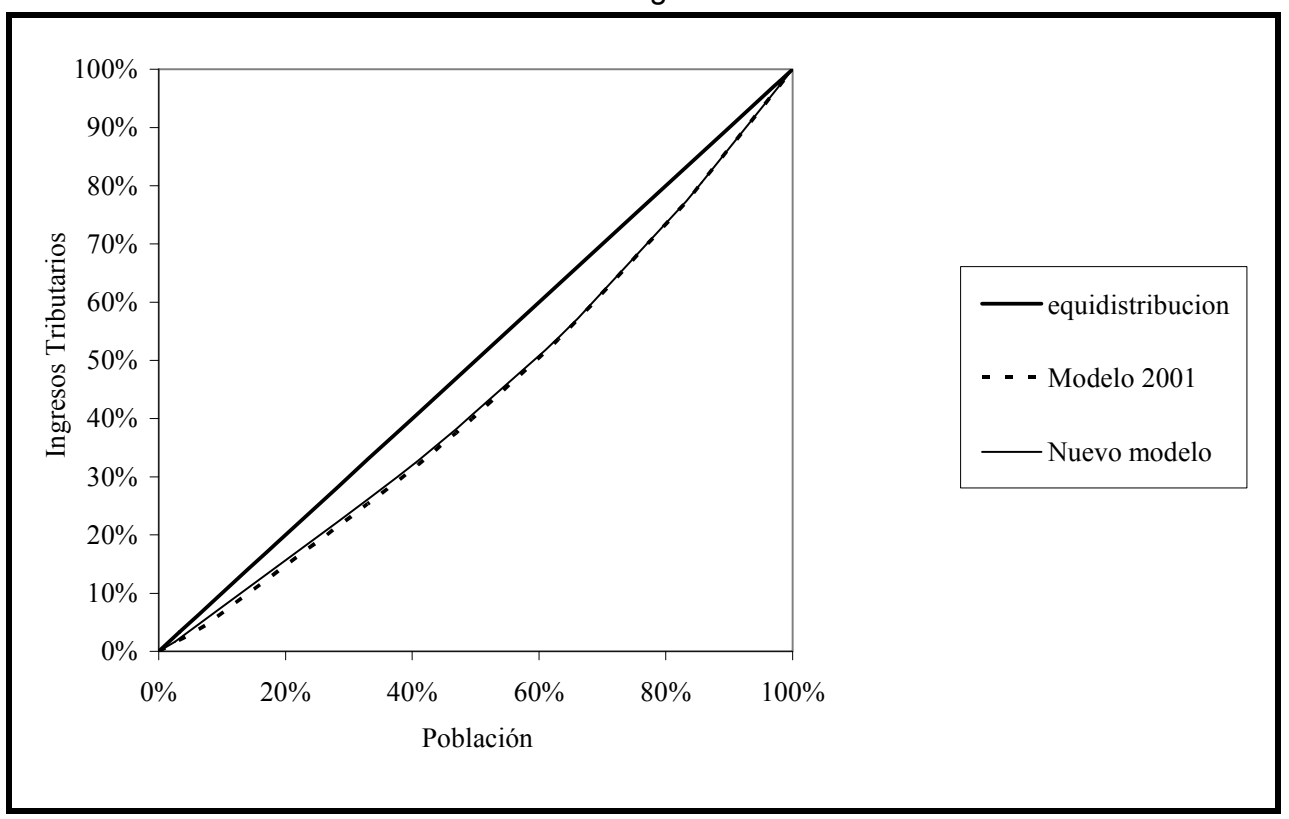

Fuente: Elaboración propia a partir de Tabla 1.

Ya en lo referido a la situación después de transferencias también los indicadores manifiestan una menor desigualdad en el nuevo modelo para el año 2007. En ambos sistemas, antiguo y nuevo, el recorte de la desigualdad es muy notable, con índices de Pechman-Okner del $70 \%$ en el nuevo modelo y de $61,1 \%$ en el modelo 2002 y de reducción relativa del coeficiente de variación del $61 \%$ y $68 \%$ respectivamente. Se puede decir, por tanto, que las transferencias del nuevo modelo tienen un mayor impacto en la reducción de la desigualdad, de casi 9 puntos porcentuales más que en el modelo 2002 en términos de índices de concentración o de 7 puntos porcentuales en términos del coeficiente de variación. Esto se debe sobre todo a que el Fondo de Garantía de Servicios Públicos Fundamentales del nuevo modelo, teniendo como objetivo nivelar las necesidades de gasto de las CC.AA., se distribuye según unos criterios de reparto, enumerados anteriormente, donde prima la población de las CC.AA. Es decir, se reparte en proporción a una especie de población ajustada, es decir, en proporción a la población ponderada por las necesidades relativas de gasto por habitante de cada C.A., lo cual, aunque no supone una igualdad estricta en términos per cápita, evidentemente sí se acerca bastante, como 
se puede observar en la Tabla 1. Teniendo en cuenta que el Fondo de Garantía de Servicios Públicos Fundamentales supone el $88,5 \%$ del total de transferencias y el $69,75 \%$ del total de financiación, es evidente que esa pauta de reparto igualatorio mencionado ejerce un peso importante en el reparto del total de financiación.

En el modelo 2002 el Fondo de Suficiencia era la transferencia que producía el impacto igualador sobre la financiación tributaria, gracias a que ejercía una función de compensación sobre la recaudación tributaria para que cada C.A. lograra alcanzar la cuantía de la restricción inicial estimada para el año base. En el nuevo modelo el Fondo de Suficiencia Global sigue teniendo esa misma función pero ya con un peso muchísimo menor, de tan sólo el $6,9 \%$ del total de transferencias y del $5,4 \%$ del total de financiación (cuando en el modelo 2002 suponía prácticamente el $100 \%$ de las transferencias y el $28,7 \%$ del total de financiación). Además, el Fondo de Suficiencia Global, con el nuevo modelo, se aplica a la cuantía agregada que por recaudación tributaria y Fondo de Garantía de Servicios Públicos Fundamentales le corresponde a cada C.A., es decir, se aplica a una base de reparto mucho más igualitaria que en el modelo 2002, que se aplicaba directamente a la recaudación tributaria.

Al respecto del impacto igualador de las transferencias también resulta importante la información que ofrece el índice de contribución por reordenación. Tal como se ha explicado, dicho índice es una medida de la pérdida de potencial igualador de las transferencias, que se produce como consecuencia de que las transferencias no sólo hacen que se igualen los ingresos per cápita sino que en muchos casos hacen que las CC.AA. que reciben menos ingresos originalmente a la postre sobrepasen a los que inicialmente obtenían más. Pues bien, se aprecia en la Tabla 2 que esa pérdida de impacto igualador se reduce con el nuevo modelo (en términos relativos desde $-63,3 \%$ en el modelo 2002 al $-46,8 \%$ en el nuevo), es decir, las transferencias del nuevo modelo producen menor desigualdad derivada de los cambios de posición que producen las mismas respecto del ranking que generan los tributos per cápita. Lo cual se debe a la mencionada pérdida de importancia cuantitativa del Fondo de Suficiencia Global en el modelo 2009. En efecto, al tener los criterios de reparto explicados, en el modelo 2002 tendía a dotar de mayores recursos per cápita a aquellas CC.AA. con menores recursos tributarios per cápita, lo cual produce lógicamente grandes cambios en posiciones en el ranking.

Por último, los indicadores hasta ahora explicados no dan información sobre el sentido de la distribución, en qué medida o no favorecen más a las CC.AA. con menor producción, es decir, del grado de progresividad de la distribución. Para cubrir tal carencia, un primer indicador utilizado es la estimación de la elasticidad renta de las transferencias. Pues bien, dicha estimación, según la Tabla 2, es estadísticamente significativa al menos a un nivel de significatividad del $10 \%$ para el modelo 2002 y al 5\% para el nuevo modelo y en ambos casos el signo del coeficiente es negativo y por tanto ambos sistemas producen resultados distributivos progresivos. Para el modelo 2002, en promedio, el hecho de que una C.A. tengan 
un $1 \%$ de PIB per cápita más que otra provoca una disminución de las transferencias per cápita percibidas del 1,4\%, y en el nuevo modelo de sólo $0,44 \%{ }^{9}$.

Por su parte, los indicadores de progresividad basados en curvas de concentración, índices de Suits y Kakwani, nos dicen, en primer lugar, que los tributos se reparten entre las CC.AA. de forma proporcional al PIB, tanto en uno como en otro modelo, como muestra unos índices tan próximos a cero (es decir, unas curvas de concentración relativa prácticamente coincidentes con la línea de equidistribución). Sin embargo, después de transferencias es evidente el gran aumento de la progresividad, que llega a situarse en $-0,136$ en el modelo 2002 y en el $-0,125$ en el nuevo modelo. Es decir, el sistema de transferencias del modelo 2002 produce un aumento de la progresividad ligeramente mayor que el del nuevo modelo, como se puede apreciar en la Figura 2.

FIGURA 2

Curvas de concentración relativa del total de financiación.

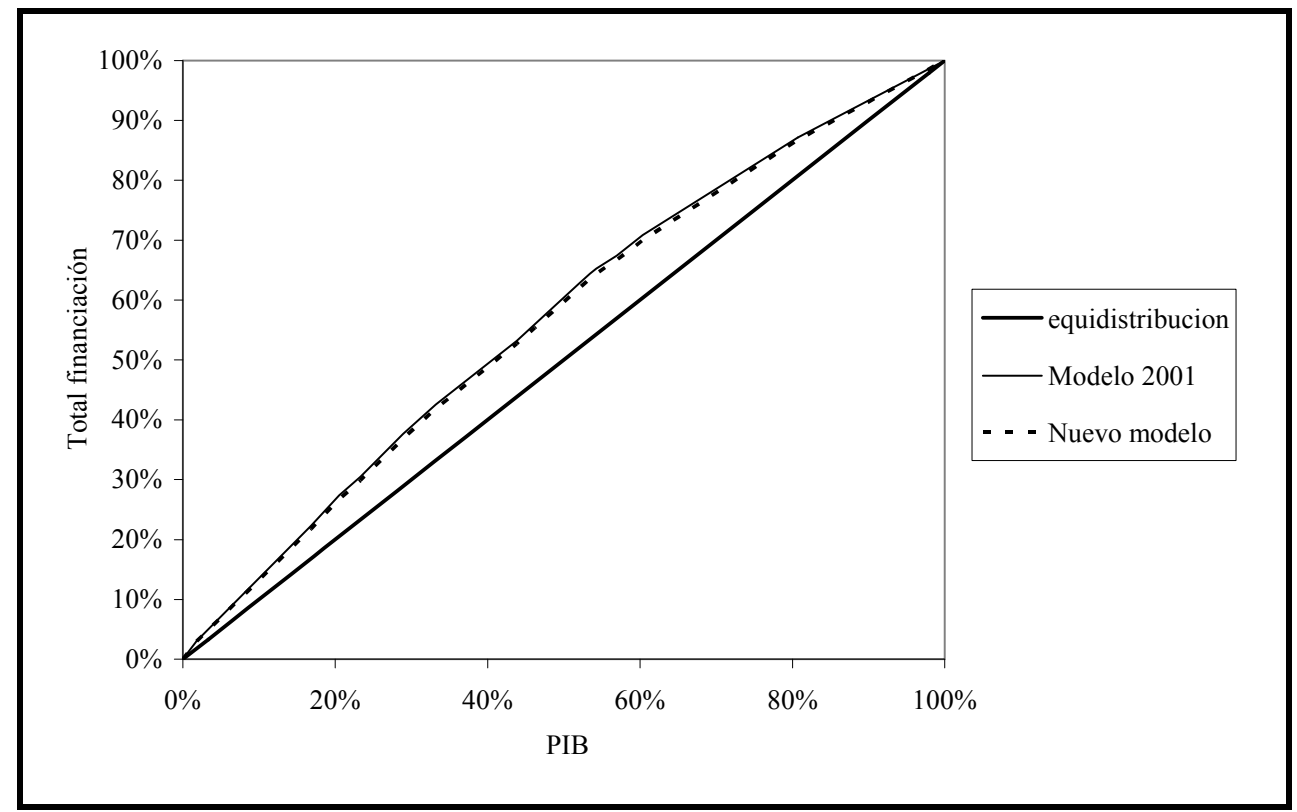

Fuente: Elaboración propia a partir de Tabla 1.

\section{CONCLUSIONES}

En este trabajo nos hemos propuesto como objetivo medir los resultados distributivos del modelo recientemente aprobado por el Consejo de Política Fiscal y Finan-

\footnotetext{
9 Téngase en cuenta que en el nuevo modelo el tamaño de las transferencias es mayor y en consecuencia la sensibilidad ante las variaciones del PIB son menores en términos relativos.
} 
ciera (Acuerdo de 15 de julio de 2009) y por la ley 22/2009 de 18 de diciembre, compararlos con los del modelo vigente desde 2002, para un año común, 2007. Creemos que esta comparación nos puede permitir conocer hasta qué punto un cambio tan drástico como el producido en la estructura del modelo tiene o no consecuencias distributivas significativas.

En primer lugar, partiendo de una ligera mayor igualdad en el reparto de los recursos tributarios, lo cual es consecuencia de los nuevos porcentajes de cesión sobre el IRPF, IVA e Impuestos Especiales, las transferencias del modelo 2009 tienen un impacto igualador mayor que el del modelo 2002, pero por una diferencia no muy significativa. En ambos modelos dicho efecto igualador es muy pronunciado. En segundo lugar, el nuevo modelo reordena menos, es decir, genera menos desigualdad por el hecho de que las CC.AA. cambien de posición en el ranking al pasar de la situación de partida de financiación tributaria a la de la financiación total, lo cual se debe al menor peso cuantitativo del Fondo de Suficiencia Global y al hecho de que éste se aplica sobre una base más igualitaria (recaudación tributaria más Fondo de Garantía de Servicios Públicos Fundamentales) en el nuevo modelo, cuando en el anterior se aplicaba directamente a los recursos tributarios de cada C.A. Por último, el grado de progresividad en el reparto de los recursos tributarios y totales es muy similar en ambos modelos, aunque algo más progresivo en el de 2002.

Ante estos resultados, la conclusión general, teniendo en cuenta las limitaciones que implica que las cifras se refieran a un año concreto, 2007, y no a una serie de años y que no sean cifras oficiales, es que el nuevo modelo no cambia sustancialmente los efectos redistributivos de las transferencias, ni en términos de desigualdad ni en términos de progresividad. Evidentemente, ello no implica que no haya variaciones en la financiación concreta de cada C.A. Lo que sí produce el nuevo modelo es menos reordenación, cuestión ésta que preocupaba a la C.A. de Cataluña hasta el punto de incluir el concepto en su nuevo Estatuto de Autonomía, aunque no en el sentido aquí analizado.

\section{REFERENCIAS BIBLIOGRÁFICAS}

ARONSON, J.R.; JONHSON, P. y LAMBERT, P.J. (1994): "Redistributive effect and unequal income tax treatment", The Economic Journal, vol. 104, n 423, pp. 262-270.

ATIENZA, P. (2003): El federalismo fiscal canadiense: comparación con el Estado de las Autonomías y posibles aplicaciones al mismo, Tesis doctoral, Málaga.

BIRD, R. y TARASOV, A.V. (2002): "Closing the gap: fiscal imbalances and intergovernmental transfers in developed federations", Working Paper 02-02, Andrew Young School of policy studies, Georgia State University.

BRODJONEGORO, B. y MARTÍNEZ-VÁZQUEZ, J. (2002): "An analysis of Indonesia's transfer system: recent perfomance and future prospects" Working Paper 02-13, Andrew Young School of policy studies, Georgia State University.

CONSEJO DE POLÍTICA FISCAL Y FINANCIERA (2009): "Acuerdo 6/2009, de 15 de julio, para la reforma del sistema de financiación de las Comunidades Autónomas de régimen común y ciudades con Estatuto de Autonomía", Madrid. 
DE LA FUENTE, A. (2009a): "El Nuevo acuerdo de financiación regional: un análisis de urgencia", Fundación de Estudios de Economía Aplicada, disponible en http://www.fedea.es/pub/est_economicos/2009/08-09.pdf (fecha de última consulta: enero 2010).

DE LA FUENTE, A. (2009b): "El Nuevo acuerdo de financiación regional: un análisis de urgencia. ADENDA", Fundación de Estudios de Economía Aplicada, disponible en http://www.fedea.es/pub/est_economicos/2009/09-09.pdf (fecha de última consulta: enero 2010).

DE LA FUENTE, A. (2009c): "Sobre la puesta en marcha del nuevo sistema de financiación regional: proyecciones para 2009 y algunas reflexiones", Fundación de Estudios de Economía Aplicada, disponible en http://www.fedea.es/pub/est_economicos/2009/ 11-09.pdf (fecha de última consulta: enero 2010).

DE LA FUENTE, A. y GUNDÍN, M. (2008): La financiación regional en España y en cuatro países federales, Fundación Caixa Galicia, CEIP.

GINI, C. (1912): "Variabilità e mutabilità, contributo allo studio delle distribuzioni e relazioni statistiche", Studi Economico-Giuridici dell' Universiti di Cagliari, 3, part. 2, pp. 1-158.

HIERRO, L.A.; ATIENZA, P. y PATIÑO, D. (2007): "Inequality and progressiveness in the distribution of revenues of the states in federal countries. A comparative study", Working Paper 07-03, International Studies Program, Andrew Young School of policy studies, Georgia State University.

HIERRO, L.A.; ATIENZA, P. y GÓMEZ-ÁLVAREZ, R. (2008): "El origen de la desigualdad y la progresividad en la distribución de la financiación de las Comunidades Autónomas de régimen común", Cuadernos de economía, n. 86, pp. 35-58.

HOFMAN, B. y CORDEIRA, S. (2004): "Ensuring interregional equity and poverty reduction", Working Paper 04-11, Andrew Young School of policy studies, Georgia State University.

KAKWANI, N.C. (1977): "Measurement of Tax Progresivity: An International Comparison", Economic Journal, $\mathrm{n}^{\circ} 87$ (345), pp. 71-80.

KAKWANI, N.C. (1984): "On Measurement of Taxes, Progressivity and Redistributive Effect of Taxes with Applications to Horizontal and Vertical Equity", Advances in Econometrics, 3, 149-168.

LAMBERT, P. (2001): The Distribution and Redistribution of Income, Manchester University Press.

MARTÍNEZ-VÁZQUEZ, J. y BOEX, J. (1999): "Fiscal decentralization in the russian federation during the transition", Working Paper 99-3, Andrew Young School of policy studies, Georgia State University.

PECHMAN, J.A. y OKNER, B. (1974): Who Bears the Tax Burden?, Brookings Institution, Washington, DC.

RAO, M.G. (2000): "Fiscal decentralization in Indian federalism", International Monetary Fund, Washington.

REYNOLDS, M. y SMOLENSKY, E. (1977): Public Expenditures, Taxes and the Distribution of Income: The United States, 1950, 1961, 1970, Academic Press, New York.

SUITS, D. B. (1977): "Measurement of Tax Progressivity", American Economic Review, vol. 67(4), pp. 747-752. 
